\title{
Event Centroiding Applied to Energy-Resolved Neutron Imaging at LANSCE
}

\author{
Nicholas P. Borges ${ }^{1, *}$, Adrian S. Losko ${ }^{2}$ (i) and Sven C. Vogel ${ }^{2}$ (it) \\ 1 Department of Physics, Worcester Polytechnic Institute, Worcester, MA 01609, USA \\ 2 Materials Science and Technology Division, Los Alamos National Laboratory, Los Alamos, NM 87545, USA; \\ alosko@lanl.gov (A.S.L.); sven@lanl.gov (S.C.V.) \\ * Correspondence: npborges@wpi.edu; Tel.: +1-781-799-2220
}

Received: 6 December 2017; Accepted: 11 February 2018; Published: 13 February 2018

\begin{abstract}
The energy-dependence of the neutron cross section provides vastly different contrast mechanisms than polychromatic neutron radiography if neutron energies can be selected for imaging applications. In recent years, energy-resolved neutron imaging (ERNI) with epi-thermal neutrons, utilizing neutron absorption resonances for contrast as well as for quantitative density measurements, was pioneered at the Flight Path 5 beam line at LANSCE and continues to be refined. Here we present event centroiding, i.e., the determination of the center-of-gravity of a detection event on an imaging detector to allow sub-pixel spatial resolution and apply it to the many frames collected for energy-resolved neutron imaging at a pulsed neutron source. While event centroiding was demonstrated at thermal neutron sources, it has not been applied to energy-resolved neutron imaging, where the energy resolution requires to be preserved, and we present a quantification of the possible resolution as a function of neutron energy. For the $55 \mu \mathrm{m}$ pixel size of the detector used for this study, we found a resolution improvement from $\sim 80 \mu \mathrm{m}$ to $\sim 22 \mu \mathrm{m}$ using pixel centroiding while fully preserving the energy resolution.
\end{abstract}

Keywords: energy-resolved neutron imaging; neutron radiography; neutron tomography; centroiding

\section{Introduction}

Material characterization by radiographic methods is driven by the contrast mechanism of the probing radiation with the sample. While $X$-rays interact with the electronic shell of the nuclei and thus provide contrast approximately proportional to the gravimetric density of a sample, neutrons interact with the nuclei and the imaging contrast has no correlation with the atomic number, but varies for thermal neutrons over orders of magnitude in cross section from isotope to isotope. This is applied routinely as an imaging modality at neutron facilities worldwide [1-4]. For many isotopes, especially heavier nuclei above $\mathrm{Zr}$, neutron absorption resonances are observed in the energy range up to a few hundred $\mathrm{eV}$, providing further contrast mechanisms. At a short pulsed neutron source, where the time-of-flight (TOF) technique for energy selection can be utilized, this can be applied to selectively map in 2D the distribution of an isotope which dominates the contrast at a certain neutron energy [5-7]. By measuring full transmission spectra, i.e., with the ability to record thousands of TOF channels in each pixel as provided by modern neutron imaging detectors [8-10] installed at modern intense pulsed neutron sources [11-15], data analysis codes such as Oak Ridge National Lab's SAMMY [16] can quantify the areal density of several isotopes measured simultaneously with the known cross sections. Based on pioneering neutron cross section measurements demonstrated at Los Alamos Neutron Center (LANSCE) [17-20], this technique has been developed over the past years at LANSCE with applications to nuclear fuels $[6,7,21]$ and scintillators [22-24]. Using tomographic reconstruction methods, this technique allows non-destructive spatially resolved measurements of isotope densities. 
In combination with the ability to characterize the same specimen with spatially resolved neutron diffraction on the High-Pressure-Preferred Orientation (HIPPO) [25] and Spectrometer for Materials Research at Temperature and Stress (SMARTS) [26] beamlines, fast neutron radiography at LANSCE's target 4 [27], proton radiography [28], and hard X-ray tomography, Los Alamos National Lab (LANL) has capabilities to provide a complete non-destructive bulk characterization on length scales ranging from atomic distances (diffraction) to the meso-scale (e.g., texture, stresses from diffraction) to dimensions, voids, and cracks as well as distributions of chemical elements and isotopes (from tomographic methods).

Spatial resolution in neutron radiography is a function of flux on sample (and thus count time), beam optics (divergence, pin hole size), and detector resolution. Many sample features, like cracks and voids, can exist with dimensions of the order of one to ten micrometers. For applications in nuclear fuels, e.g., characterization of fuel pellets within rodlets for irradiation testing, non-destructive measurements of the fuel to cladding distance is of great interest, with a typical distance being $71 \pm 5 \mu \mathrm{m}$. Deviations from this distance can affect the temperature profile of the assembly and thus the outcome of an irradiation test or fuel burn-up in a power reactor. Accurate measurement of this distance therefore defines a resolution requirement for tomographic methods to be applied to nuclear fuels and a goal for the efforts at LANSCE supporting these programs. Approaches to improve the resolution while leaving all other parameters approximately constant are of great interest since exposure time at neutron sources is limited. Event centroiding, where the center of gravity of a detection event covering multiple pixels on an imaging detector is determined to sub-pixel resolution, is such a method. While pixel or event centroiding has been applied to neutron imaging at continuous wave neutron sources such as PSI [29] or NIST [30], it has not been demonstrated for energy-resolved neutron imaging at a pulsed neutron source where thousands of radiographs are acquired for each neutron pulse. Current methods of neutron centroiding involve charge pulse mode with the detector, where each individual neutron supplies a charge distribution in the event and can distinguish between overlapping events. The problem with this technique is that energy resolved measurements are sacrificed [29]. The method described here preserves the ability to measure e.g., fuel-cladding distances using cold and thermal neutrons while simultaneously measure isotope distributions with higher neutron energies. Here, we describe our implementation of event centroiding in the MediPix-based pixilated neutron TOF imaging detector as well as a quantitative assessment of the achieved resolution improvement.

\section{Materials and Methods}

Imaging measurements described in this report were performed using Flight Path 5 (FP5) at the Lujan Neutron Scattering Center (for a description see M. Mocko et al. [31]). The spallation neutron pulses are created by $270 \mathrm{~ns}$ long $800 \mathrm{MeV} \mathrm{H}+$ pulses impinging on a tungsten target. The neutrons are then moderated using liquid water at room temperature and collimated using 2.5 to $5 \mathrm{~cm}$ thick steel and polyethylene disks with round holes of diameters increasing from $2 \mathrm{~cm}$ to $5 \mathrm{~cm}$ from $4.5 \mathrm{~m}$ to $6 \mathrm{~m}$ distance from the moderator. At $6 \mathrm{~m}$ from the moderator, additional $20 \mathrm{~cm}$ of steel and poly disks with round holes of $0.5 \mathrm{~cm}$ are used to further collimate the beam for high resolution measurements (Figure 1). The resulting spectrum for neutrons at the Lujan center is shown in Figure 2. It provides a thermal neutron flux of $\sim 2.4 \times 10^{7} \mathrm{n} \cdot \mathrm{cm}^{-2} \cdot \mathrm{s}^{-1}$ at $\sim 8.8 \mathrm{~m}$ from the moderator with a peak at $25 \mathrm{meV}$ [32].

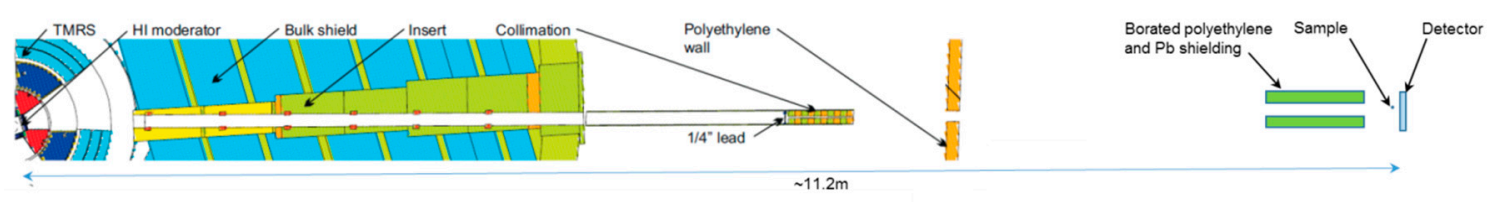

Figure 1. Flight Path 5 Schematic. Note that the polyethylene walls and the borated polyethylene and $\mathrm{Pb}$ shielding were not used for the measurements reported here. 


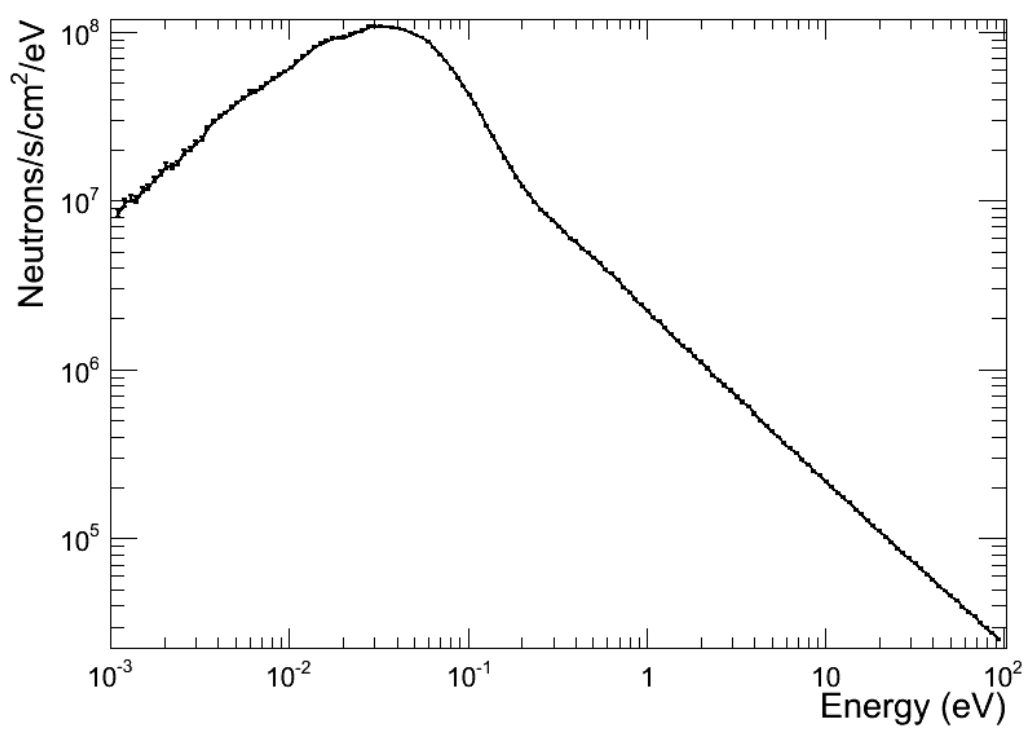

Figure 2. The total neutron energy spectra produced by the Lujan Center. The spectra were calculated with the maximum proton beam currents of $125 \mu$ a for the Lujan Center [31].

For the measurements reported here, the detector was positioned $\sim 11.2 \mathrm{~m}$ from the water moderator. The test patterns to measure the resolution were produced by imaging a gadolinium (Gd) grating fabricated at the Paul Scherrer Institute, Villigen, Switzerland [33]. The resolution grating was placed as close as possible to the detector to minimize blur from beam divergence. A collimator (smallest diameter) to sample distance of about $L=5 \mathrm{~m}$ and smallest beam diameter of $\mathrm{D}=0.5 \mathrm{~cm}$ resulted in a collimation ratio $\mathrm{L} / \mathrm{D}$ of 1000 . With a sample to detector distance $\mathrm{d}$ of $\sim 1 \mathrm{~cm}$, the geometric blur is given by $\mathrm{d} /(\mathrm{L} / \mathrm{D}) \approx 10 \mu \mathrm{m}$ (note that this blur is not the same as the spatial resolution). As an internal standard for the resonance measurements and position calibration of the detector, a $0.1 \mathrm{~mm}$ thick Ta sheet was placed in front of the sample (source side). To reduce background from gamma radiation emitted from the spallation target, $\sim 12 \mathrm{~mm}$ lead was also placed into the beam at the exit of the incident collimator pipe.

The pixilated neutron TOF imaging detector used at LANL is custom-designed and manufactured and shown in Figure 3. It consists of a Gd/B doped microchannel plate (MCP) and MediPix read-out chip $[34,35]$. To account for differences in the individual pixel response of the detector, an open-beam measurement was recorded over the entire energy range $(0.5 \mathrm{meV}$ to $200 \mathrm{eV})$. Individual TOF images are divided by the normalized open-beam image. The pixel size of the detector is $55 \mu \mathrm{m}$ with an active area of $512 \times 512$ pixels i.e., $28.16 \times 28.16 \mathrm{~mm}^{2}$. The Medipix MCP detector at the LANSCE facility allows for single neutron event detection due to the high efficiency (50-70\% for thermal neutrons) [10] and has a $100 \mathrm{kHz}$ frame rate in single particle mode. The Medipix detector views a MCP doped with boron and gadolinium and coupled with another stack of MCPs to provide amplification. The size of the channels for the doped MCPs are $8 \mu \mathrm{m}$ and the amplifiers have $10 \mu \mathrm{m}$ channels. The detector sensor utilizes a complementary metal-oxide-semiconductor (CMOS) chip with four $256 \times 256$ arrays of pixels that are $55 \mu \mathrm{m}^{2}$ pixels. The detector is held under vacuum and provides energy resolution by the TOF method with the supply of a time zero event trigger pulse, generated by the proton pulse immediately before hitting the spallation target.

Centroiding has been used in astronomy applications since 1980 [36] to achieve sub-pixel high resolution and converting this technique to neutron imaging is relatively new [29]. In general, multiple pixels are activated when a neutron impinges on an imaging detector. The location of the neutron can be calculated by computing the center of mass or centroid of the activation distribution. In the case of the MediPix based pixilated neutron TOF detector used here, each $55 \mu \mathrm{m}$ pixel is divided into $5 \times 5=25$ sub-pixels, resulting in an increase of the effective resolution to $55 \mu \mathrm{m} / 5=11 \mu \mathrm{m}$. 
One complexity with centroiding arises from the case when a double or triple interaction with multiple neutrons overlapping on one frame occurs. In that case, the center of mass cannot be distinguished and the calculation will result in a center of mass resultant from the overlapping events. However, in pulse charge mode it is possible to distinguish up to 50\% overlap [29]. To avoid this occurrence for this work, the acquisition time was short while additionally negating any event that is greater than 20 pixels or less than 2 pixels, with acquisition time, minimum, and maximum pixel area being adjustable parameters. A single neutron event can be seen in Figure 4, with the zoomed in portion (Figure $4 \mathrm{~b}$ ) signifying the division and centroided location (Figure 4c) for one particle. The highest spatial resolution achievable by this method is limited by the MCP's channel size $(11 \mu \mathrm{m})$. Due to the cascade effect within the microchannels, neutrons absorbed at different locations within the same channel cannot be discriminated by this method.

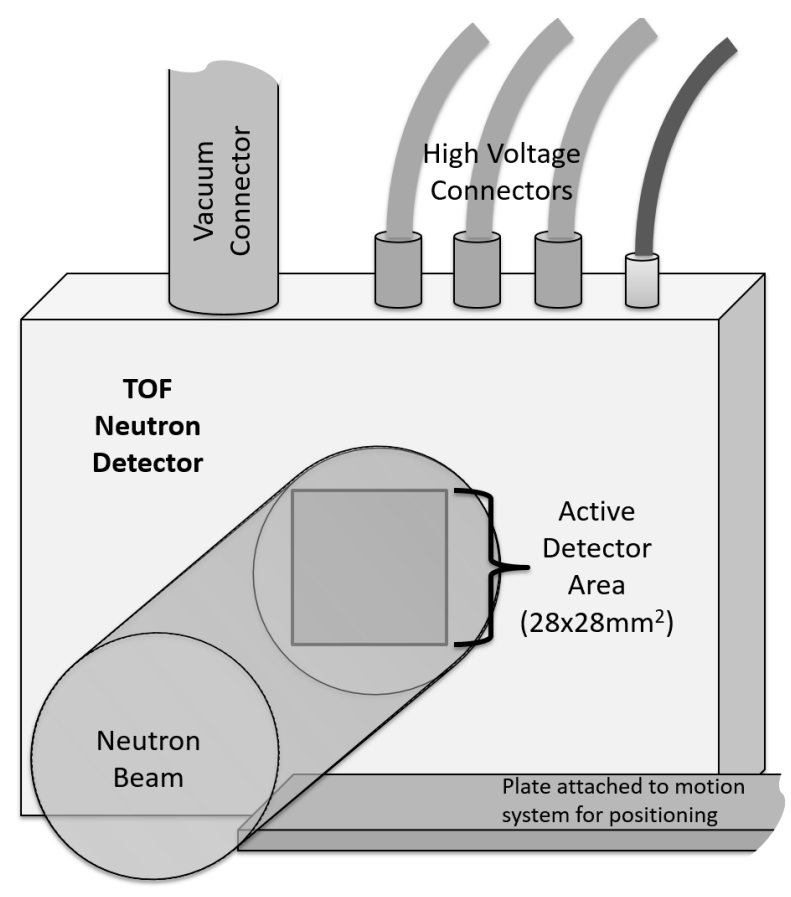

Figure 3. Detector schematic.

Single particle events in integration mode do not provide a charge distribution across the activated pixels [29], instead each activated pixel is incremented by a value of one. A simple non-intensity weighted centroiding process in normal integration mode will produce results slightly less accurate as compared to utilizing a Gaussian fit for a charged activation area in pulse mode. An acquisition time of $180 \mathrm{~s}$ allowed for an acceptable ratio of interacting neutrons and data save and processing time (360 s) while avoiding overlapping events. Unprocessed data is stored on the hard-drive and processed in a sequential order. Single neutron events can be observed within a single TOF frame before the 3197 individual time bins are summed together for a non-energy resolved image. The centroiding process can allow for the creation of general bins; cold neutrons $(6 \mathrm{meV}-25 \mathrm{meV})$, thermal neutrons ( $25 \mathrm{meV}-960 \mathrm{meV}$ ), and epithermal neutrons $(960 \mathrm{meV}-242 \mathrm{eV}$ ) or leave the individual 3197 bins for resonance analysis. The method of event centroiding is utilized by running a python script in conjunction with the detector's native pixelman software. The python script takes a saved fits file from pixelman and runs a secondary macro written for ImageJ that can centroid and convert the image to a tabulated text file for compressing larger data files. The general bin centroided method is presented and the advantages are discussed. 


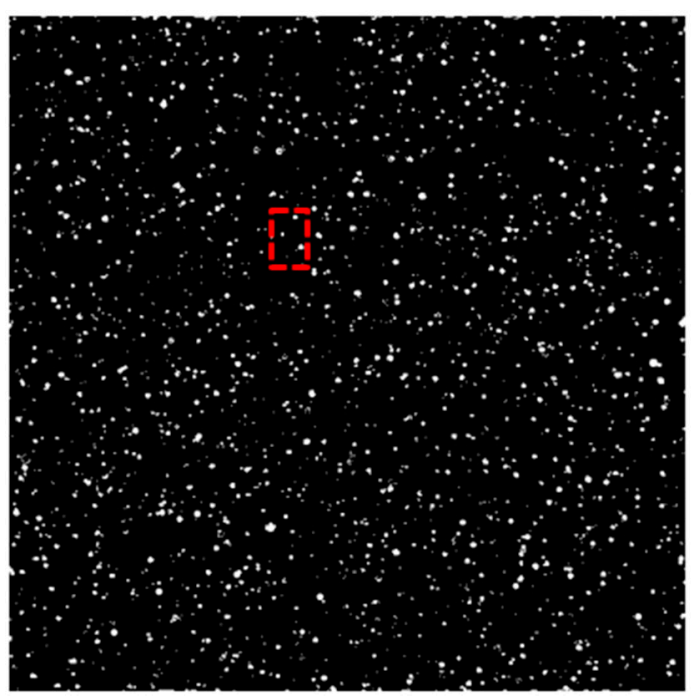

(a)

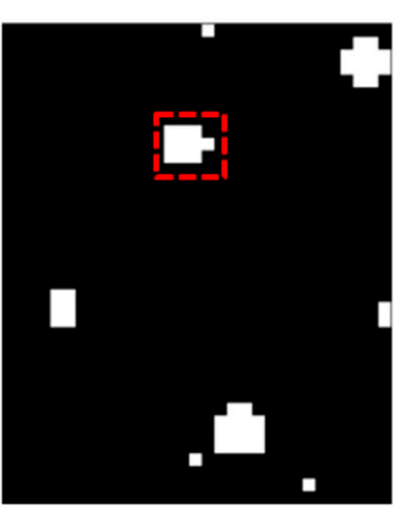

(b)

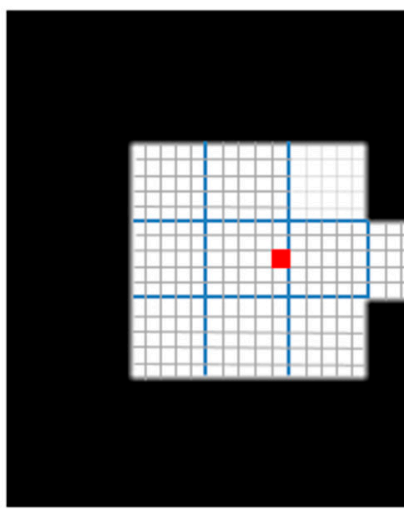

(c)

Figure 4. Neutron TOF image for the cold neutron radiograph energy region showing individual events(a). Zoomed in section of (a) marked by red dashed rectangle (b). Illustrated sub-pixel break down for a single neutron event marked by red dashed area in (c). One single $55 \mu \mathrm{m}$ pixel is converted into a $5 \times 5$ subpixel array with sizes of $11 \mu \mathrm{m}$ each. The red square indicates where the event will be centroided to a single sub pixel.

An open beam measurement to see the detector response with $12+\mathrm{h}$ of acquisition time reveals that the centroiding introduces some artefacts (Figure 5). The grid in the Fourier transform, Figure 5b, of the centroided open beam image, Figure $5 \mathrm{a}$, is indicative of a roughly $13 \mu \mathrm{m}$ pattern that is consistently present throughout the entire radiograph whereas in Figure $5 c$, the Fourier transform of the same open beam image without centroiding, the pattern is not present. This visible pattern from centroiding is regarded as an artefact but it does not degrade the overall image. The most likely source of this artefact is produced when creating the $25 \times 25$ subpixels per pixel, this pattern typically happens when the centroiding program has a slight bias to the middle of the subpixels than the edges (can be seen in the zoomed region in Figure 5a). Whereas, the point in the middle of Fourier transform is an undefined point in marking the zero point and any frequency pattern is projected radially from that point. This high resolution open beam image is used for normalizing and correcting radiographs described below.
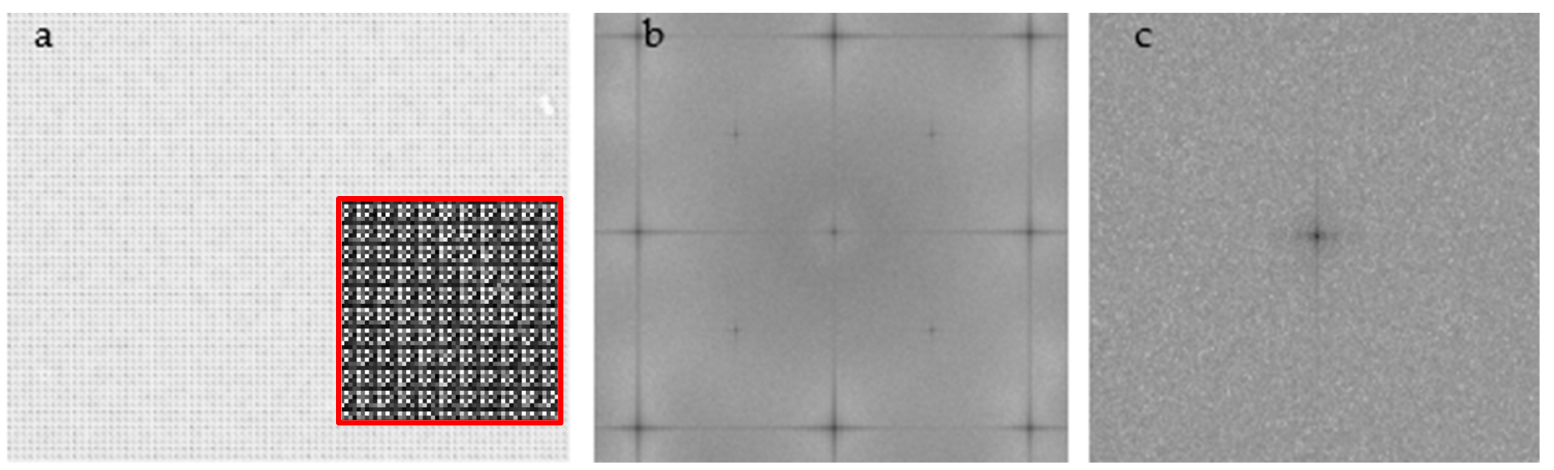

Figure 5. Centroided image with $11 \mu \mathrm{m}$ effective pixel size for open beam radiograph. The $800 \%$ zoomed-in section to show centroiding pattern effect is in the bottom right corner (a). Fast Fourier transform of the centroided open beam area to demonstrate the detector characteristics which reveals some artefacts from centroiding (b). Fast Fourier transform of non-centroided open beam demonstrating there are no unusual artefacts in the raw data (c). 
The fast Fourier transform (Figure 5b,c) converts the image into the frequency domain from the spatial domain. For example, a radiograph of $200 \mu \mathrm{m}$ line pairs $(400 \mu \mathrm{m}$ period) on a detector with a $50 \mu \mathrm{m}$ pixel pitch would result in a peak intensity at 8 pixels/period. If the line pairs are resolved, this would indicate a spatial-resolution of at least $200 \mu \mathrm{m}$.

\section{Results}

The Paul Scherrer Institut (PSI) resolution grating [33,37] (Figure 6) is an ideal standard for evaluating the overall resolution of an instrument setup (see reference for comparable construction of similar grating produced by PSI). The frequency of line pairs which are resolved gives a direct correlation to the overall image resolution. The grating is comprised of gadolinium regions of lines with widths of $160 \mu \mathrm{m}, 80 \mu \mathrm{m}, 40 \mu \mathrm{m}, 20 \mu \mathrm{m}, 10 \mu \mathrm{m}$, and $5 \mu \mathrm{m}$. Here, the PSI grating was placed directly on the face of the detector enclosure $(12 \mathrm{~mm}$ from detector face to active area) and the image was acquired at 30 seconds intervals with 1093 iterations (total of $9 \mathrm{~h}$ of exposures). A non-centroided image demonstrates the previous resolution limitation of the Medipix detector system.

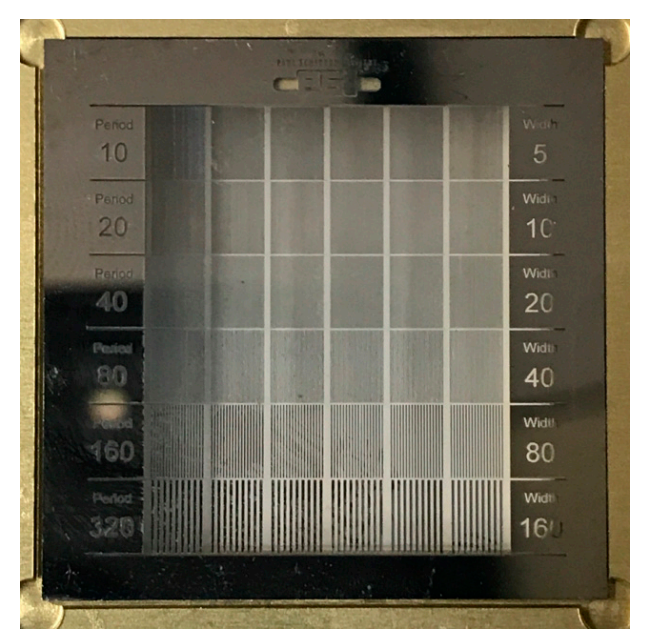

(a)

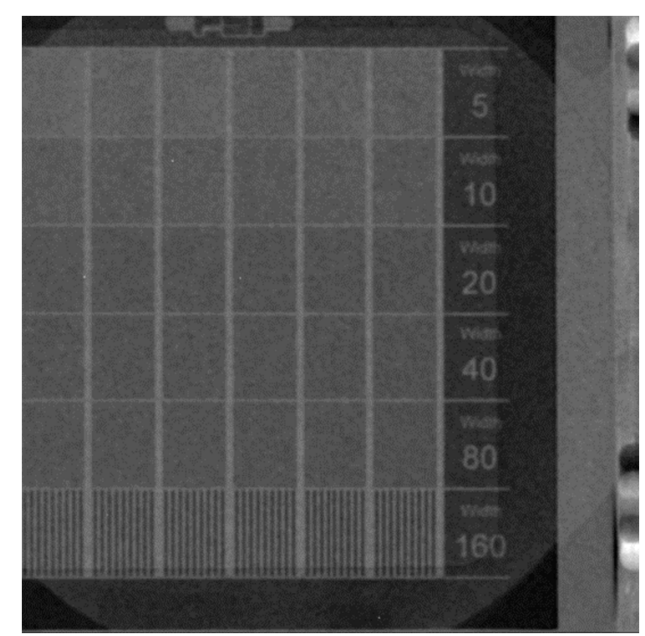

(b)

Figure 6. Photograph of PSI resolution grating (a). Non-centroided neutron image of the PSI resolution grating, comprised of $\mathrm{SiO}_{2}$ glass backing and $5 \mu \mathrm{m}$ thick $\mathrm{Gd}$ absorber with laser-etched test patterns (b) $[33,37]$.

The non-centroided PSI grating (Figure 7a) was compared to the energy resolved centroided image as shown in Figure 7. The radiograph was adjusted with open beam and scaled to represent percentage of neutrons transmitted through the sample. A 9-h exposure provides sufficient statistics to separate the $20 \mu \mathrm{m}$ width line pairs with cold neutrons (Figure 7c). The thermal neutron energy radiograph (Figure $7 \mathrm{~d}$ ) can resolve the $20 \mu \mathrm{m}$ width line pairs whereas the contrast for epithermal neutrons suffers due to lower absorption cross section of $\mathrm{Gd}$, i.e., the grating becomes transparent (Figure 7e). The contrast is weaker for the centroided image of all energies (Figure $7 \mathrm{~b}$ ) resembling a white neutron beam weighed by the moderator spectrum, illustrating the benefit of energy-resolved neutron imaging to pick the energy range with the best contrast for a given problem.

Figure 8 illustrates multiple fast Fourier transform peaks on a single scatter plot. The peaks have been converted to correspond from pixel/cycle to resolution using the width pairs on the PSI grating. The centroided fast Fourier transform peaks are visible to the $20 \mu \mathrm{m}$ limit regardless of energy dependence for the processing. The original unprocessed radiograph compared to the centroided counterpart cannot resolve lower than the $80 \mu \mathrm{m}$ peak whereas the centroided image can resolve $20 \mu \mathrm{m}$. 


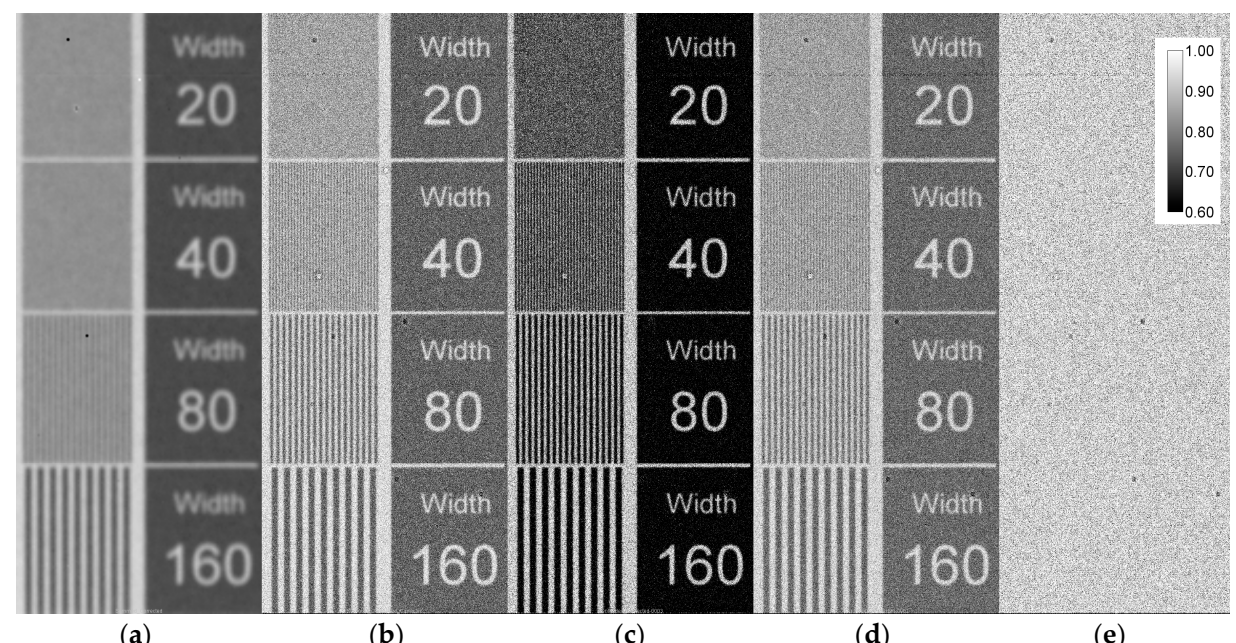

(a)

(b)

(c)

(d)

(e)

Figure 7. Radiograph of PSI grating for original $55 \mu \mathrm{m}$ original pixel size (a). Centroided image with $11 \mu \mathrm{m}$ effective pixel size for entire energy range of neutrons summed together $(\mathbf{b})$ Centroided image with $11 \mu \mathrm{m}$ effective pixel size for cold (c), thermal (d), and epithermal (e) neutrons.

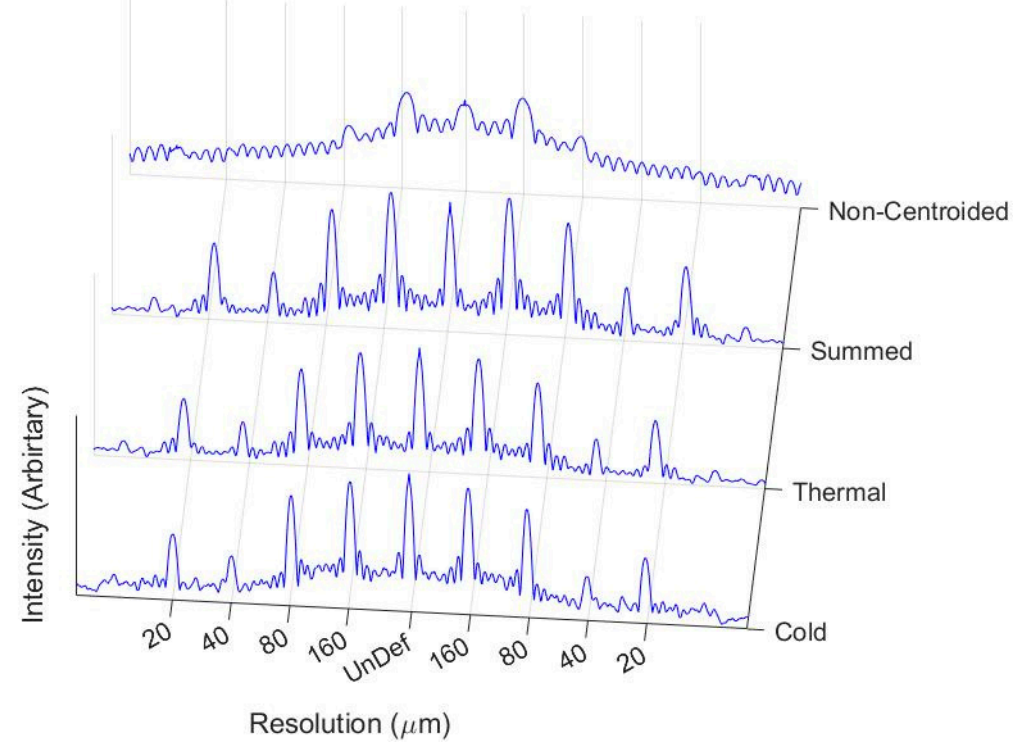

Figure 8. The fast Fourier transform for centroided cold, thermal, non-energy resolved, and raw non-centroided radiographs.

To investigate the resolution as a function of neutron energy, finer energy bins were processed, as shown in Figure 9, ranging from $6 \mathrm{meV}$ to $100 \mathrm{meV}$. For each energy bin, the contrast resolution was calculated. The contrast resolution $(C)$ is defined as the signal ratio between the dark $\left(S_{d}\right)$ and light $\left(S_{l}\right)$ regions resulting from line width pairs on the grating [38]. Here, the $80 \mu \mathrm{m}$ line width pairs were chosen, due to the non-centroided radiograph not resolving the $40 \mu \mathrm{m}$ line width pairs.

$$
C=\frac{\left(S_{d}-S_{l}\right)}{\left(S_{d}+S_{l}\right)}
$$

While contrast resolution is not strictly equivalent to spatial resolution, it is a good indicator of the ability to measure the differences in intensities that define features such as edges in a radiograph, thus quantifying the quality of the image. With increasing neutron energy, the cross section for the gadolinium decreases, resulting in the contrast ratio decreasing to the point where the grating is no 
longer visible (red marker limit on graph). The centroided image has a higher contrast ratio when compared to the non-centroided image over the entire energy spectrum.

The trend of the decrease in contrast resolution with increasing energy correlates directly with the neutron cross section of gadolinium, suggesting that the contrast resolution is dependent on the sample material only. To illustrate this point, the boron neutron cross section, representing the detector efficiency, is also overlaid in Figure 9. The boron that is inherent in the MCP does not correlate between the contrast resolution of the sample and the boron cross section.

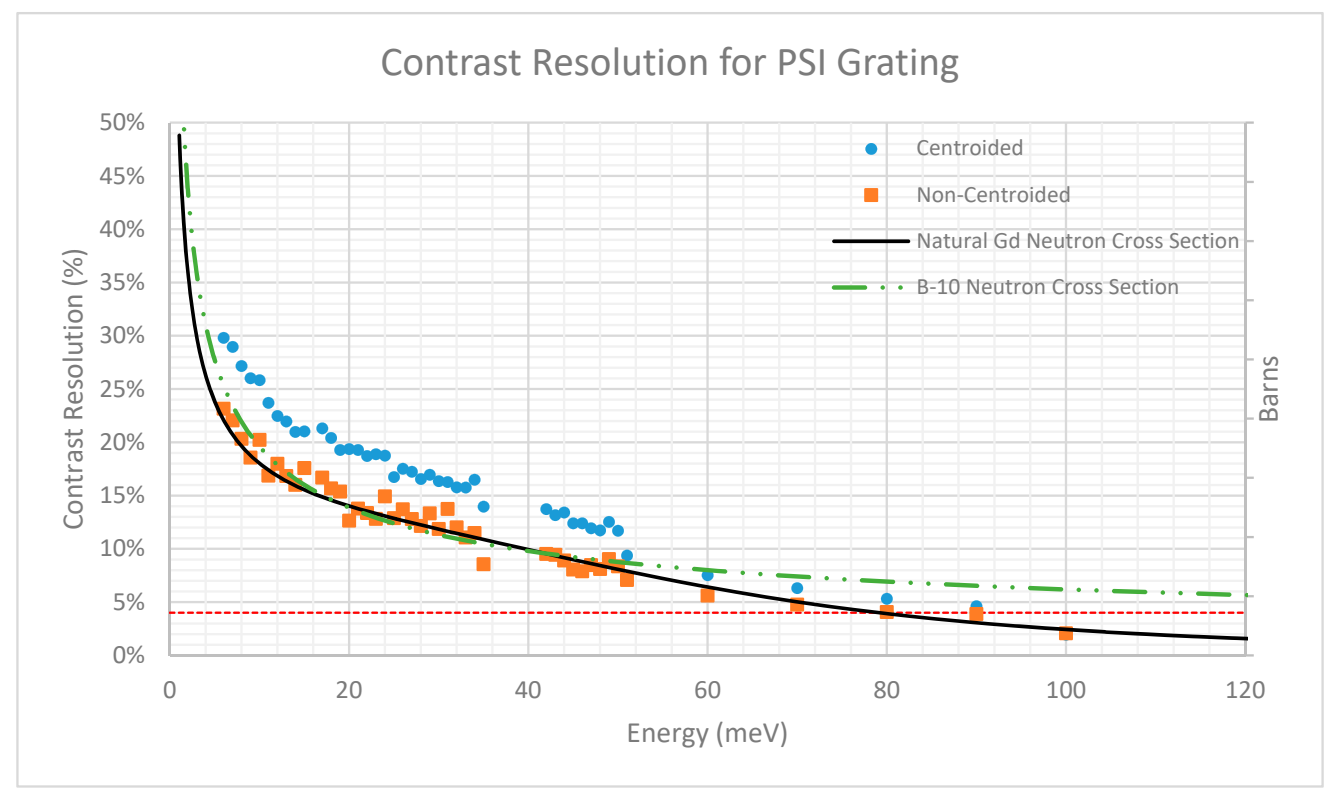

Figure 9. The contrast resolution for finer energy bins ranging from $6 \mathrm{meV}$ to $100 \mathrm{meV}$. The grating is no longer visible at a contrast of $4 \%$ (dashed red). The Gd (solid black) neutron cross sections are compared to the trend of the contrast resolution.

The highest possible spatial resolution of the system, assuming perfect counting statistics, can be found by taking the modulation transfer function (MTF) at 10\% of a slant edge function. The American Section of the International Association for Testing Materials (ASTM) standard E545 provides information on characterizing neutron beam and image quality by imaging a lead disk, boron nitride disk, and a cadmium wire setup [39]. The design of the imaging standard is reproduced in Figure 10.

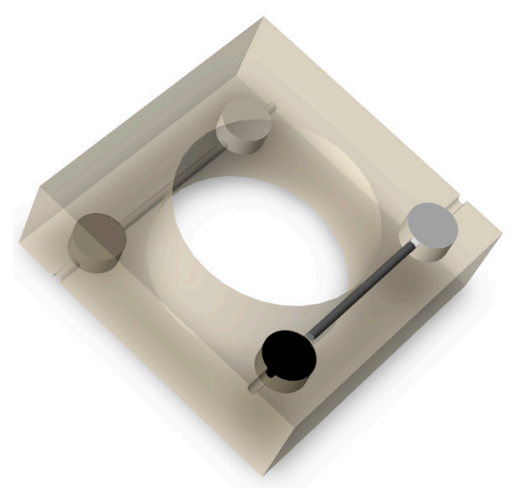

Figure 10. Association for Testing Materials (ASTM) E 2003 standard services after 3d modelling that consists of polytetrafluoroethylene body, 99.9\% cadmium wire, 99.9\% lead disk (black), and 40\% elemental boron nitride disk (white) [40]. 
The MTF of an imaging system is defined by the absolute value of its optical transfer function and is limited at the high end by the Nyquist frequency [41]. The limiting spatial resolution is considered to be when the MTF crosses $10 \%$ since a single sinusoidal wave needs two adjacent pixels to display a full cycle. The Nyquist frequency is then always 0.5 cycles per pixel (or twice the size of one pixel). To obtain the MTF, the cadmium wire provides an edge to produce an edge spread function. The edge spread function is differentiated to obtain the line spread function. The MTF is calculated by taking the Fourier transform of the line spread function. The standard non-centroided and energy resolved centroided radiographs of the ASTM standard object are shown in Figure 11. The processed radiographs confirm a resolution in agreement with the determination with the PSI grating. The MTF at 10\% shows that the resolution of the radiographs could be further increased with better counting statistics.

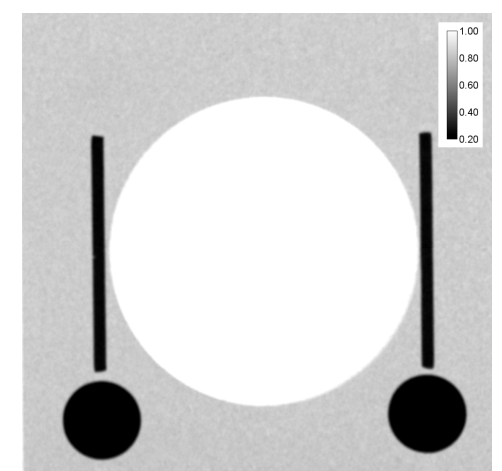

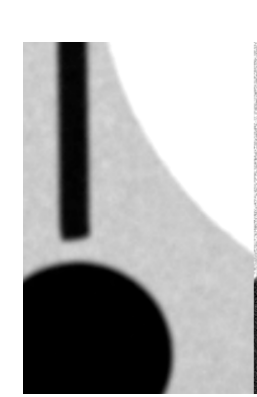

(b)

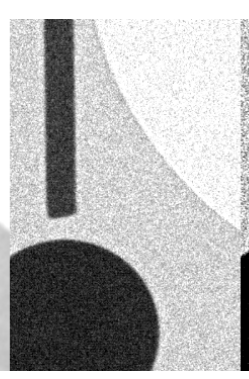

(c)

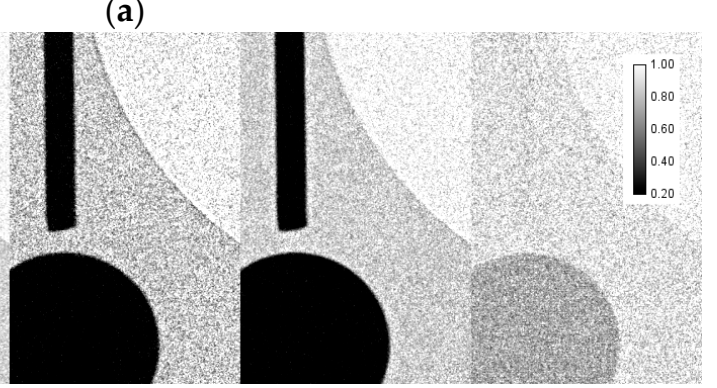

(d) (e) (f)

Figure 11. Non-centroided image with original $55 \mu \mathrm{m}$ pixel size imaging ASTM beam purity standard with entire spectrum of neutrons, resulting in $\sim 80 \mu \mathrm{m}$ resolution $(\mathbf{a}, \mathbf{b})$. Centroided image with $11 \mu \mathrm{m}$ effective pixel size for entire energy range of neutrons summed together (c). Centroided image with $11 \mu \mathrm{m}$ effective pixel size and with $\sim 40 \mu \mathrm{m}$ spatial resolution for each cold (d), thermal (e), and epithermal (f) neutrons. The cadmium wire at the epi-thermal energy is almost completely transparent, signifying a decrease in neutron cross section in the higher energy regime.

The MTF is plotted vs spatial frequency for the ASTM standard radiographs (Figure 12). To obtain resolution in $\mu \mathrm{m}$, cycles/pixel must be converted to line pairs $/ \mathrm{mm}$ first by using the pixel pitch size. The resolution can now be determined by converting line pairs $/ \mathrm{mm}$ to $\mu \mathrm{m}$ at $10 \%$ on the MTF curve. The non-centroided radiograph results in the highest possible resolution of $70 \mu \mathrm{m}$, consistent with the results of the PSI grating where the $80 \mu \mathrm{m}$ width pairs are barely resolved. The cold neutron energy centroided region results in a resolution of $29 \mu \mathrm{m}$ and the thermal region results in $22 \mu \mathrm{m}$. Using the measured data and sub-pixel size of $11 \mu \mathrm{m}$, this cannot be any higher at this point due to the Nyquist frequency being the size of two pixels, or $22 \mu \mathrm{m}$. Epithermal resolution was not calculated due to low statistics and more importantly the lack of attenuation from the gadolinium precluding a reliable calculation of the edge spread function. An element with a higher cross section in the 
epithermal energy region to provide an edge is needed to quantify spatial resolution in the epithermal energy range.

The original non-centroided radiograph does not conform to the traditional Nyquist frequency rule-of-thumb (twice the pixel size of $55 \mu \mathrm{m}$ ) due to charge overlap between pixels and can give a resolution below the Nyquist frequency which was determined with the PSI grating to be $80 \mu \mathrm{m}$. Since the non-energy resolved radiograph is just the cold, thermal, and epithermal summed together, the resolution for the MTF is degraded by the addition of the epithermal regime. The MTF provides a more exact resolution at which the image can be resolved when compared to the PSI grating. The PSI grating only has very coarse step wedges and there is not an adequate edge for measuring the edge spread function. For the ASTM standard, the thermal range has a better resolution than the cold range due to the penetration of the neutrons through the material which is not seen with the PSI grating. The Gd in the grating effectively blocks all neutrons within the thermal and cold region, whereas, with the standard introduces a scattering medium of polytetrafluoroethylene.

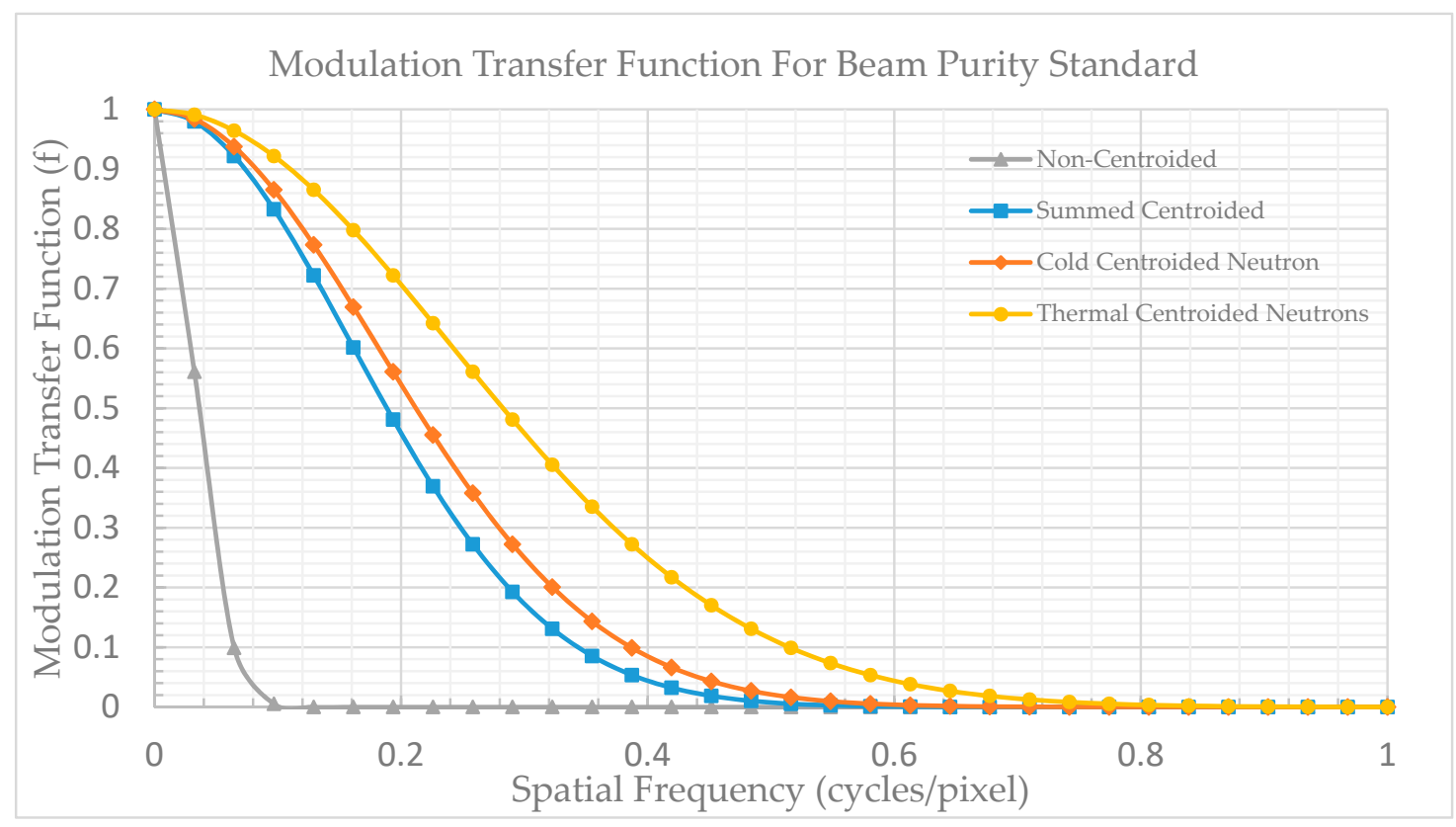

Figure 12. Modulation Transfer Function plots for non-centroided, centroided cold, thermal, and non-energy resolved centroided radiographs.

\section{Discussion}

Imaging with higher number of subpixels will allow the Nyquist frequency limit to go down and provide higher resolution images. Applying centroiding improves overall resolution, but is limited by the incident neutron flux and in the case of MCP detectors, by the channel size. One solution would be to increase the number of iterations for each image, but the centroiding pattern effect, visible in the inset of Figure 5a, would only get worse with more subpixels. With proper software and hardware implementation, event centroiding can be conducted in parallel with the data acquisition, i.e., resolution improvements occur without increases in beam time. Spatial resolution was improved by a factor of $\sim 4$ using event centroiding compared to the non-centroided results, implying a similar resolution improvement for tomography. For the ASTM standard, the spatial resolution was better for the thermal neutrons versus the cold neutrons, while the test object did not provide enough contrast for the epithermal neutrons. With the current characterization samples, based on gadolinium contrast, we were able to quantify the contrast resolution as a function of energy. The contrast resolution increased by $7 \%$ with the centroiding process. The energy dependence scaled with the gadolinium cross section allowing to conclude that the contrast resolution mostly depends on the sample material 
and not on incident flux or detector properties. In the near future, the method will be applied to nuclear fuel pellets to attempt the demonstration of the improved spatial resolution for isotope quantification. This will also allow us to assess the spatial and contrast resolution for a larger energy range.

Acknowledgments: This work has benefited from the use of the Lujan Neutron Scattering Center at LANL, which is operated by Los Alamos National Security LLC under DOE contract DEAC5206NA25396. We acknowledge support from the U.S. Department of Energy, Nuclear Technology Research and Development Program, Advanced Fuels Campaign. NPB appreciates funding from the Seaborg Institute at LANL. SV and NPB acknowledge funding from DOE/NNSA NA-22 for funding of project LB15-V-GammaDetMater-PD3Jfu.

Author Contributions: Nicholas P. Borges and Adrian S. Losko conceived and designed the experiments, performed the experiments, and analyzed the data. Sven C. Vogel supervised the work and provided the PSI grating. All authors contributed to writing the paper.

Conflicts of Interest: The authors declare no conflict of interest.

\section{References}

1. Lehmann, E.H. Neutron Imaging Methods and Applications in Neutron Applications in Earth, Energy and Environmental Sciences; Springer: Berlin, Germany, 2009; pp. 319-348.

2. Strobl, M.; Manke, I.; Kardjilov, N.; Hilger, A.; Dawson, M.; Banhart, J. Advances in neutron radiography and tomography. J. Phys. D 2009, 42, 243001. [CrossRef]

3. Kardjilov, N.; Manke, I.; Hilger, A.; Strobl, M.; Banhart, J. Neutron imaging in materials science. Mater. Today 2011, 14, 248-256. [CrossRef]

4. Lehmann, E.H.; Grunzweig, C.; Peetermans, S.; Kaestner, A.; Mannes, D. Advances in Neutron Radiology for Nondestructive Testing. Mater. Eval. 2014, 72, 437-442.

5. Schillebeeckx, P.; Borella, A.; Emiliani, F.; Gorini, G.; Kockelmann, W.; Kopecky, S.; Lampoudis, C.; Moxon, M.; Cippo, E.P.; Postma, H.; et al. Neutron resonance spectroscopy for the characterization of materials and objects. J. Instrum. 2012, 7, C03009. [CrossRef]

6. Tremsin, A.S.; Vogel, S.C.; Mocko, M.; Bourke, M.A.; Yuan, V.; Nelson, R.O.; Brown, D.W.; Feller, W.B. Non-destructive studies of fuel pellets by neutron resonance absorption radiography and thermal neutron radiography. J. Nucl. Mater. 2013, 440, 633-646. [CrossRef]

7. Tremsin, A.S.; Losko, A.S.; Vogel, S.C.; Byler, D.D.; McClellan, K.J.; Bourke, M.A.; Vallerga, J.V. Non-contact measurement of partial gas pressure and distribution of elemental composition using energy-resolved neutron imaging. AIP Adv. 2017, 7, 015315. [CrossRef]

8. Tremsin, A.S.; Feller, W.B.; Downing, R.G. Efficiency optimization of microchannel plate (MCP) neutron imaging detectors. I. Square channels with 10 B doping. Nucl. Instrum. Methods Phys. Res. 2005, 539, $278-311$. [CrossRef]

9. Siegmund, O.H.; Vallerga, J.V.; Tremsin, A.S.; Mcphate, J.; Feller, B. High spatial resolution neutron sensing microchannel plate detectors. Nucl. Instrum. Methods Phys. Res. 2007, 576, 178-182. [CrossRef]

10. Tremsin, A.S.; McPhate, J.B.; Vallerga, J.V.; Siegmund, O.H.; Feller, W.B.; Lehmann, E.; Dawson, M. Improved efficiency of high resolution thermal and cold neutron imaging. Nucl. Instrum. Methods Phys. Res. 2011, 628, 415-418. [CrossRef]

11. Tremsin, A.S.; McPhate, J.B.; Kockelmann, W.A.; Vallerga, J.V.; Siegmund, O.H.; Feller, W.B. Energy-resolving neutron transmission radiography at the ISIS pulsed spallation source with a high-resolution neutron counting detector. IEEE Trans. Nucl. Sci. 2009, 56, 2931-2937. [CrossRef]

12. Kockelmann, W.; Zhang, S.Y.; Kelleher, J.F.; Nightingale, J.B.; Burca, G.; James, J.A. IMAT—A new imaging and diffraction instrument at ISIS. Phys. Procedia 2013, 43, 100-110. [CrossRef]

13. Tremsin, A.S.; Vogel, S.C.; Mocko, M.; Bourke, M.A.; Yuan, V.; Nelson, R.O.; Brown, D.W.; Feller, W.B. Energy resolved neutron radiography at LANSCE pulsed neutron facility. Neutron News 2013, 24, 28-32. [CrossRef]

14. Tremsin, A.S.; Shinohara, T.; Kai, T.; Ooi, M.; Kamiyama, T.; Kiyanagi, Y.; Shiota, Y.; McPhate, J.B.; Vallerga, J.V.; Siegmund, O.H.; et al. Neutron resonance transmission spectroscopy with high spatial and energy resolution at the J-PARC pulsed neutron source. Nucl. Instrum. Methods Phys. Res. 2014, 746, 47-58. [CrossRef] 
15. Bilheux, H.Z.; Bilheux, J.C.; Bailey, W.B.; Keener, W.S.; Davis, L.E.; Herwig, K.W.; Cekanova, M. Neutron imaging at the Oak Ridge National Laboratory: Application to biological research. In Proceedings of the 2014 Annual Oak Ridge National Laboratory Biomedical Science and Engineering Center Conference (BSEC), Oak Ridge, TN, USA, 6-8 May 2014.

16. Larson, N.M. Updated User's Guide for Sammy: Multilevel R-Matrix Fits to Neutron Data Using Bayes' Equations; Oak Ridge National Laboratory (ORNL): Oak Ridge, TN, USA, 2008.

17. Lynn, J.E.; Trela, W.J. Resonance neutron methods for determining statistical properties of phonon spectra. Nucl. Instrum. Methods Phys. Res. 1996, 108, 147-158. [CrossRef]

18. Lynn, J.E.; Kwei, G.H.; Trela, W.J.; Yuan, V.W.; Cort, B.; Martinez, R.J.; Vigil, F.A. Vibrational properties of $\mathrm{Pu}$ and $\mathrm{Ga}$ in a Pu-Ga alloy from neutron-resonance Doppler spectroscopy. Phys. Rev. B. 1998, $58,11408$. [CrossRef]

19. Lynn, J.E.; Trela, W.J.; Meggers, K. Neutron Doppler broadening studies of tantalum and tungsten metal. Nucl. Instrum. Methods Phys. Res. 2002, 192, 318-330. [CrossRef]

20. Yuan, V.W.; Bowman, J.D.; Funk, D.J.; Morgan, G.L.; Rabie, R.L.; Ragan, C.E.; Quintana, J.P.; Stacy, H.L. Shock temperature measurement using neutron resonance spectroscopy. Phys. Rev. Lett. 2005, 94, 125504. [CrossRef] [PubMed]

21. Vogel, S.C.; Losko, A.S.; Pokharel, R.; Ickes, T.L.; Hunter, J.F.; Brown, D.W.; Voit, S.L.; Tremsin, A.S.; Bourke, M.A.; McClellan, K.J. Non-Destructive Preirradiation Assessment of UN/U-Si "LANL1" ATF Formulation; Los Alamos National Lab: Los Alamos, NM, USA, 2016.

22. Tremsin, A.S.; Makowska, M.G.; Perrodin, D.; Shalapska, T.; Khodyuk, I.V.; Trtik, P.; Boillat, P.; Vogel, S.C.; Losko, A.S.; Strobl, M.; et al. In situ diagnostics of the crystal-growth process through neutron imaging: Application to scintillators. J. Appl. Crystallogr. 2016, 49, 743-755. [CrossRef] [PubMed]

23. Tremsin, A.S.; Perrodin, D.; Losko, A.S.; Vogel, S.C.; Shinohara, T.; Oikawa, K.; Peterson, J.H.; Zhang, C.; Derby, J.J.; Zlokapa, A.M.; et al. In-situ Observation of Phase Separation During Growth of Cs2LiLaBr6: Ce Crystals Using Energy-Resolved Neutron Imaging. Cryst. Growth Des. 2017, 17, 6372-6381. [CrossRef]

24. Tremsin, A.S.; Perrodin, D.; Losko, A.S.; Vogel, S.C.; Bourke, M.A.; Bizarri, G.A.; Bourret, E.D. Real-time Crystal Growth Visualization and Quantification by Energy-Resolved Neutron Imaging. Sci. Rep. 2017, 7, 46275. [CrossRef] [PubMed]

25. Wenk, H.R.; Lutterotti, L.; Vogel, S. Texture analysis with the new HIPPO TOF diffractometer. Nucl. Instrum. Methods Phys. Res. 2003, 515, 575-588. [CrossRef]

26. Bourke, M.A.; Dunand, D.C.; Ustundag, E. SMARTS-A spectrometer for strain measurement in engineering materials. Appl. Phys. A 2002, 74, S1707-S1709. [CrossRef]

27. Bergmann, B.; Nelson, R.O.; O’Donnell, J.M.; Pospisil, S.; Solc, J.; Takai, H.; Vykydal, Z. Time-of-flight measurement of fast neutrons with Timepix detectors. J. Instrum. 2014, 9, C05048. [CrossRef]

28. King, N.S.; Ables, E.; Adams, K.; Alrick, K.R.; Amann, J.F.; Balzar, S.; Barnes, P.D., Jr.; Crow, M.L.; Cushing, S.B.; Eddleman, J.C.; et al. An $800-\mathrm{MeV}$ proton radiography facility for dynamic experiments. Nucl. Instrum. Methods Phys. Res. 1999, 424, 84-91. [CrossRef]

29. Tremsin, A.S.; McPhate, J.B.; Vallerga, J.V.; Siegmund, O.H.; Feller, W.B.; Lehmann, E.; Kaestner, A.; Boillat, P.; Panzner, T.; Filges, U. Neutron radiography with sub-15 $\mu \mathrm{m}$ resolution through event centroiding. Nucl. Instrum. Methods Phys. Res. 2012, 688, 32-40. [CrossRef]

30. Hussey, D.S.; LaManna, J.M.; Baltic, E.; Jacobson, D.L. Neutron imaging detector with $2 \mu \mathrm{m}$ spatial resolution based on event reconstruction of neutron capture in gadolinium oxysulfide scintillators. Nucl. Instrum. Methods Phys. Res. 2017, 866, 9-12. [CrossRef]

31. Mocko, M.; Muhrer, G.; Tovesson, F. Advantages and limitations of nuclear physics experiments at an ISIS-class spallation neutron source. Nucl. Instrum. Methods Phys. Res. 2008, 589, 455-464. [CrossRef]

32. Ino, T.; Ooi, M.; Kiyanagi, Y.; Kasugai, Y.; Maekawa, F.; Takada, H.; Muhrer, G.; Pitcher, E.J.; Russell, G.J. Measurement of neutron beam characteristics at the Manuel Lujan Jr. neutron scattering center. Nucl. Instrum. Methods Phys. Res. 2004, 525, 496-510. [CrossRef]

33. Grünzweig, C.; Frei, G.; Lehmann, E.; Kühne, G.; David, C. Highly absorbing gadolinium test device to characterize the performance of neutron imaging detector systems. Rev. Sci. Instrum. 2007, 78, 053708. [CrossRef] [PubMed]

34. Siegmund, O.H.; Vallerga, J.V.; Tremsin, A.S.; Feller, W.B. High spatial and temporal resolution neutron imaging with microchannel plate detectors. IEEE Trans. Nucl. Sci. 2009, 56, 1203-1209. [CrossRef] 
35. Tremsin, A.S. High resolution neutron counting detectors with microchannel plates and their applications in neutron radiography, diffraction and resonance absorption imaging. Neutron News 2012, 23, 35-38. [CrossRef]

36. Dennison, E.W.; Stanton, R.H. Ultra-Precise Star Tracking Using Charge Coupled Devices (CCDs). In Proceedings of the SPIE 0252 Smart Sensors, San Diego, CA, USA, 1 January 1980.

37. Kaestner, A.P.; Kis, Z.; Radebe, M.J.; Mannes, D.; Hovind, J.; Grünzweig, C.; Kardjilov, N.; Lehmann, E.H. Samples to Determine the Resolution of Neutron Radiography and Tomography. Phys. Procedia 2017, 88, 258-265. [CrossRef]

38. Hendrick, R.E.; Raff, U.; Stark, D.D.; Bradley, W.G. Magnetic Resonance Imaging, 2nd ed.; Mosby Year Book: St. Louis, MO, USA, 1992; pp. 109-144.

39. STM E545-14. Standard Test Method for Determining Image Quality in Direct Thermal Neutron Radiographic Examination; ASTM International: West Conshohocken, PA, USA, 2014.

40. Nray Services Inc. 56A Head Street Dundas Ontario; Nray Services Inc.: Dundas, ON, Canada.

41. Neitzel, U.; Egbert, B.; Gerhard, H.; Paul, R. Determination of the modulation transfer function using the edge method: Influence of scattered radiation. Med. Phys. 2004, 31, 3485-3491. [CrossRef] [PubMed]

(C) 2018 by the authors. Licensee MDPI, Basel, Switzerland. This article is an open access article distributed under the terms and conditions of the Creative Commons Attribution (CC BY) license (http:/ / creativecommons.org/licenses/by/4.0/). 